\title{
Le créationnisme est-il seulement une affaire de
} foi ?

Enquête sur l'origine des espèces en France et au Maroc

Is creationism only a matter of faith? The origin of species in France and in Morocco

Dominique Guillo

\section{(2) OpenEdition}

\section{Journals}

Édition électronique

URL : http://journals.openedition.org/ress/3001

DOI : 10.4000/ress.3001

ISSN : 1663-4446

Éditeur

Librairie Droz

Édition imprimée

Date de publication : 15 mai 2015

Pagination : 227-263

ISSN : 0048-8046

Référence électronique

Dominique Guillo, «Le créationnisme est-il seulement une affaire de foi ? », Revue européenne des sciences sociales [En ligne], 53-1 | 2015, mis en ligne le 01 janvier 2019, consulté le 22 avril 2019. URL http://journals.openedition.org/ress/3001; DOI : 10.4000/ress.3001 


\title{
LE CRÉATIONNISME EST-IL SEULEMENT UNE AFFAIRE DE FOI? ENQUÊTE SUR L'ORIGINE DES ESPĖCES EN FRANCE ET AU MAROC
}

DOMINIQUE GUILLO

CNRS, Paris - GEMASS et CRESC (UM6P), Rabat

dominiqueguillo@yahoo.fr

\begin{abstract}
Résumé. Le rejet du darwinisme, l'adhésion à une représentation de l'origine des espèces marquée par le créationnisme et la foi religieuse sont considérés dans beaucoup d'enquêtes comme des attitudes étroitement liées l'une à l'autre. L'objectif du présent article est de montrer que ce schéma explicatif doit être révisé. Les représentations des espèces vivantes n'obéissent pas à une logique binaire fondée sur l'opposition entre foi et raison, religion et science. Pour le montrer, nous présentons ici une comparaison entre deux terrains a priori fort contrastés: la France et le Maroc. Cette enquête fait également ressortir une caractéristique majeure de ces croyances, négligée dans les investigations sur ce thème: elles sont souvent intrinsèquement floues, parce que beaucoup d'individus ont vis-à-vis de cette question - l'origine des espèces - une forme d'indifférence. Cette thématique ne paraît donc pas universellement et nécessairement investie d'une puissante charge sacrée et polémique, même si elle l'est parfois très fortement.
\end{abstract}

Mots-clés: animaux, biologie ordinaire, cognition, créationnisme, darwinisme, évolutionnisme, foi, islam, rationalité, religion, sécularisation.

\begin{abstract}
Rejection of Darwinism, creationism and religious faith are seen in many investigations in the social sciences as attitudes closely related to each other. The purpose of this paper is to show that this explanatory schema is wrong. Ordinary representations of living species do not obey a binary logic based on the opposition between faith and reason, religion and science. To show this, I present a comparison between two fields of investigation: the first, in France; the second, in Morocco. Furthermore, this inquiry underlines a major feature of these beliefs : they are often inherently blurred, because many individuals exhibit towards this issue - the origin of species - a form of indifference. Therefore, this question does not appear to be a matter universally and necessarily strongly considered as sacred, even if it is sometimes the case.
\end{abstract}

Keywords: animals, cognition, creationism, darwinism, evolutionism, faith, folkbiology, islam, rationality, religion, secularization. 
La réception du darwinisme ${ }^{1}$ est aujourd'hui très largement analysée au moyen d'une grille de lecture dont les lignes essentielles ramènent à la thèse wébérienne de la sécularisation opérée par la science. En affirmant que l'émergence et le devenir des espèces vivantes ne doivent rien à l'action intentionnelle de quelque force occulte, le darwinisme, fait-on valoir, a contribué au « désenchantement du monde» provoqué par le rationalisme moderne, en l'étendant au domaine biologique ${ }^{2}$. Il n’a pu manquer, ce faisant, de rencontrer la résistance des mouvements religieux, et plus largement des croyants. Réciproquement, il a contribué de manière décisive à diffuser auprès des noncroyants - ou des croyants moins fervents - une conception de la nature débarrassée des schèmes créationnistes.

Telle est en particulier la thèse qui supporte une bonne partie des travaux actuels en philosophie, en sciences cognitives et en sciences sociales. On retrouve dans ces travaux un schéma explicatif récurrent, articulé autour d'arguments tantôt logiques, tantôt empiriques, qui visent à soutenir l'hypothèse d'une opposition irréconciliable entre deux manières contradictoires de bâtir une représentation de la nature vivante. La première est guidée par l'esprit scientifique, la seconde par des raisonnements rendus fallacieux par la foi, à travers les schèmes cognitifs qu'elle conduit les individus à mobiliser (voir, par exemple, Dennett, 2007 ; Dawkins, 2006 ; Deniz et al. 2008 ; Kim et Nehm, 20I I).

| Nous emploierons le terme «darwinisme» pour désigner ici l'ensemble des propositions qui font aujourd'hui globalement consensus en biologie de l'évolution (voir Mayr, 200l et voir infra), et qui trouvent leur origine, pour une très large part, dans L'Origine des espèces (Darwin, 1992 [1859]): sur ce dernier point, voir également Mayr, 200 I, ou encore Gayon, 1992.

2 On trouve chez Freud une célèbre explication de la réception du darwinisme - celui-ci aurait infligé une «blessure narcissique» à l'être humain et à la religion - qui conduit à une assez semblable conclusion, bien que les arguments soient quelque peu différents de ceux que l'on trouve classiquement dans les thèses de la sécularisation (Freud, 1985 [1911]). 
En simplifiant, cette thèse générale prédit que, plus on sera croyant, plus on affichera des convictions créationnistes, plus on aura une représentation de l'origine des espèces éloignée de la science et marquée par les schèmes créationnistes ${ }^{3}$, plus on invoquera des arguments d’autorité religieux, ou la foi, plus on sera hostile au darwinisme, plus cette hostilité sera véhémente et, enfin, plus on répugnera à admettre un lien généalogique entre l'homme et le singe. Inversement, moins on sera croyant, plus on affichera une adhésion au darwinisme, plus on aura une représentation des espèces vivantes proche de ce que dit aujourd'hui la biologie à propos de l'origine des espèces, plus on invoquera les faits, la science et la raison pour appuyer cette représentation de la nature vivante, plus on sera hostile au créationnisme et plus cette hostilité sera véhémente.

À première vue, cette thèse paraît confirmée par la vive hostilité affichée par de nombreux mouvements religieux à l'égard du darwinisme, mouvements religieux chrétiens - comme les néo-évangélistes américains - aussi bien que musulmans - à l'exemple de ceux qui se sont cristallisés ces dernières années autour du turc Harun Yahya et de son Atlas of Creation (2006), largement diffusé en Europe. Réciproquement, le succès de la théorie de l'évolution dans l'espace public d'un pays laïque comme la France, où il n'est presque jamais contesté, parait ajouter un argument supplémentaire en faveur de cette thèse.

Les recherches qui conduisent à de telles conclusions se concentrent, toutefois, très souvent, sur les acteurs directement et activement impliqués dans les débats publics qui entourent le créationnisme, à savoir les religieux, les scientifiques, les enseignants ou encore les étudiants en biologie. Pourtant, dès lors que l'on examine le contenu des croyances ordinaires, plutôt que les convictions affichées par ces acteurs, le tableau qui se dessine est beaucoup plus complexe, parfois même très différent. Tels sont du moins les enseignements qui ressortent d'enquêtes que nous avons menées - et que nous pour-

3 Comme on le verra plus loin, il est essentiel de distinguer l'adhésion affichée au créationnisme, d'une part, et le fait d'avoir une représentation de l'origine des espèces marquée par le créationnisme, d'autre part. Un individu peut, en effet, avoir une représentation marquée - à son insu - par le créationnisme, tout en affichant une adhésion au «darwinisme» et un rejet du «créationnisme». 
suivons ${ }^{4}$ - dans deux contextes a priori fort contrastés, notamment par la place qu'ils accordent à la religion : la France, d'une part, et le $\mathrm{Maroc}^{5}$, d'autre part.

Tels qu'ils se donnent à voir sur ces terrains, les liens qui s'établissent dans l'esprit des acteurs ordinaires entre la «science», les croyances relatives à la «nature vivante » et la «foi religieuse » n'ont rien d'automatique et échappent aux binarités dans lesquelles la thèse de la sécularisation - nous l'appellerons ainsi pour simplifier - tend à les enfermer. Deux éléments, négligés dans la plupart des enquêtes sur ce thème, contribuent à complexifier considérablement ces liens et le jeu des représentations et des positionnements sur cette question. Tout d'abord, de fait, la représentation du «darwinisme » affichée par les acteurs ordinaires, qu'ils adhèrent à cette doctrine ou qu'ils la rejettent, est le plus souvent très éloignée de ce que mettent les scientifiques spécialisés en biologie de l'évolution sous le même mot. Par ailleurs, beaucoup d'individus semblent manifester pour la question de l'origine des espèces un manque d'intérêt et d'engagement, une forme d'indifférence, qui donnent aux croyances que l'on peut leur prêter un flou intrinsèque. Cette indifférence et ce flou passent inaperçus dans la plupart des enquêtes. Il est pourtant capital d'en tenir compte, comme on va le voir, pour dessiner la logique qui commande les représentations et les conflits sur ces questions.

4 Ces enquêtes sont actuellement prolongées dans le cadre du programme ANR LICORNES.

5 Sur la réception du darwinisme en contexte musulman, voir également les conclusions originales de Koning, 2006. 


\section{LA FRANCE:}

\section{UN «ÉVOLUTIONNISME» TRÈS ÉLOIGNÉ DU DARWINISME}

\section{I.I. UN PROBLÈME POSÉ À UN ÉCHANTILLON D'ÉTUDIANTS D'UNE GRANDE ÉCOLE SCIENTIFIQUE}

Pour tenter d'éclairer cette logique, nous avons analysé plusieurs corpus en France $^{6}$. Nous insisterons plus particulièrement, dans le cadre du présent article, sur l'un de ces corpus, qui nous a servi de trame principale pour notre comparaison avec le Maroc (voir deuxième partie). Ce corpus est constitué des réponses fournies par un échantillon d'étudiants scientifiques à un problème que nous leur avons posé. Ce problème était formulé de la façon suivante. Sur une première page figuraient six photos, sans ordre net apparent. Ces photos représentaient respectivement un «singe», un «humain», une «amibe», un «chêne», un «lézard» et une «baleine». Ces noms étaient écrits en légende sous chacune des photos. Sur une seconde page, l'on demandait aux étudiants : «tracez l’arbre généalogique qui relie selon vous ces individus les uns aux autres».

L’objectif principal de cette expérience était de faire ressortir, autant que possible, les lignes essentielles des conceptions de l'origine des espèces propres aux personnes interrogées. Plus spécifiquement, dans le cas français, nous voulions déterminer à quel degré et sur quels points les schémas manifestent une proximité avec la conception darwinienne de la généalogie des êtres vivants. Nous voulions voir, en particulier, si l'on retrouve sans ambiguïté dans ces schémas les ruptures radicales opérées par le darwinisme avec cinq schèmes qui, à l'opposé, jouent un rôle central dans les représentations créationnistes de l'origine des espèces : le fixisme, ou si l'on veut l'hypothèse d'une absence de lien généalogique entre individus d'espèces différentes; l'échelle des êtres anthropocentrée - c'est-à-dire couronnée par l'être humain; l'absence de rôle accordé au hasard; la conception essentialiste des espèces et de l'ordre que leur classification dessine; et, enfin, la téléologie, conséquence nécessaire de chacun des quatre

6 Les autres terrains, ou corpus, sont: le courrier envoyé à la revue Sciences \& Vie par des lecteurs en réaction à un numéro sur l'évolution; un échantillon de manuels scolaires des années 1990; et, enfin, des entretiens menés auprès de professeurs des écoles à propos de l'évolution (Guillo, 2015). Nous évoquerons ce dernier corpus à la fin de cette partie. 
schèmes précédents (voir Mayr, 200I). Ces cinq schèmes jouent un rôle central dans les représentations créationnistes parce qu'ils impliquent tous que des entités extra-mondaines - qu'elles prennent la forme d'un dieu personnifié, d'entités métaphysiques ou d'un ordre abstrait sous-jacent - sont intervenus dans la formation des espèces.

Dans l'enquête que nous exposons ici, le problème a été posé à I 20 étudiants ( $\mathrm{N}=$ I20) de troisième année d'une grande école scientifique française ${ }^{7}$. Sur cet échantillon, les résultats se sont avérés fortement convergents.

\section{I.2. UN TRANSFORMISME MARQUÉ PAR L'ÉCHELLE DES ÊTRES ANTHROPOCENTRÉE}

Premier résultat important, aucun étudiant n’a proposé une représentation fixiste des espèces vivantes, même partielle: dans les schémas recueillis, aucune espèce n’a été laissée sans lien avec les autres. Parmi les izo étudiants interrogés, I04 sur I 20 - soit 86,7\%, - ont proposé un schéma généalogique (Tableau I). Un étudiant a répondu qu'il n’avait aucun avis sur la question. I5 n’ont donné aucune réponse (Tableau I).

Tableau I. Fixisme ou transformisme

\begin{tabular}{|l|c|c|c|c|c|}
\hline & NON-RÉPONSES & $\begin{array}{c}\text { AVEU } \\
\text { D'IGNORANCE }\end{array}$ & SCHÉMAS FIXISTES & $\begin{array}{c}\text { SCHÉMAS } \\
\text { TRANSFORMISTES }\end{array}$ & TOTAL \\
\hline $\begin{array}{l}\text { VALEUR } \\
\text { ABSOLUE }\end{array}$ & 15 & 1 & 0 & 104 & 120 \\
\hline$\%$ & 12,5 & 0,8 & 0 & 86,7 & 100 \\
\hline
\end{tabular}

7 Ces étudiants ont suivi après le baccalauréat pour la grande majorité d'entre eux des classes préparatoires scientifiques de deux ou trois années («mathématiques spéciales» et «mathématiques supérieures»). Une fraction minoritaire a suivi des classes préparatoires «BL» (sciences économiques et sociales) ou des cursus de statistiques et d'économie à l'Université. Durant ces formations, et durant leur scolarité dans cette école - qui forme des ingénieurs statisticiens - ces étudiants n'ont pas suivi de cours spécifiquement consacré à la biologie. Toutefois, la théorie de l'évolution leur a été exposée dans le détail au cours de leur Terminale (la très grande majorité de ces étudiants ont suivi la filière «S») et dans le cours d'introduction aux sciences sociales qui leur a été dispensé deux mois auparavant dans cette école. 
La représentation des espèces que donnent à voir ces schémas est donc transformiste, c'est-à-dire, sur ce point, proche du darwinisme et éloignée de nombre de représentations qui s’affichent sous la bannière du créationnisme.

Cette absence totale de schéma fixiste, dans un échantillon d'étudiants d'un pays où la laïcité joue un rôle capital, doit-elle pour autant conduire à valider la thèse de la sécularisation? Pour répondre à cette question, il est essentiel de souligner, comme on l'a évoqué plus haut, que le fixisme n’est pas le seul schème constitutif des représentations créationnistes de la nature. Or, si l'on regarde de plus près ces différents schémas, on constate qu'en réalité la version de la transformation - ou de l'évolution - des espèces qu'ils manifestent renferme trois autres schèmes qui occupent généralement une place centrale dans les représentations créationnistes des espèces.

Tout d'abord, l'échelle des êtres anthropocentrée commande en profondeur les différents schémas proposés, sous des formes plus ou moins apparentes. Les plus patentes sont la linéarisation du dispositif graphique - les êtres sont reliés deux à deux par des lignes directes, plutôt que par des embranchements ou des chevrons figurant des «ancêtres communs »-, d'une part, et le couronnement de ces schémas par l'être humain, d'autre part, qui traduit la signification hiérarchique et scalaire d'un ordre linéaire manifestement gradué selon le degré de complexité prêté aux êtres.

La linéarisation du schéma et, à travers elle, le rôle régulateur joué par le schème de l'échelle des êtres anthropocentrée, sont, par exemple, tout à fait explicites dans le schéma suivant (Schéma I). 
Schéma I.

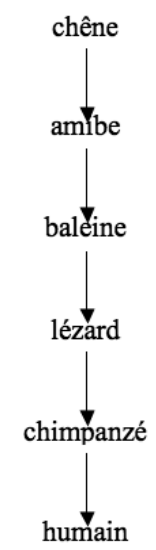

Ces thématiques sont également présentes, malgré les apparences, dans les schémas non linéaires, arborescents, semblables au suivant (Schéma 2).

Schéma 2.

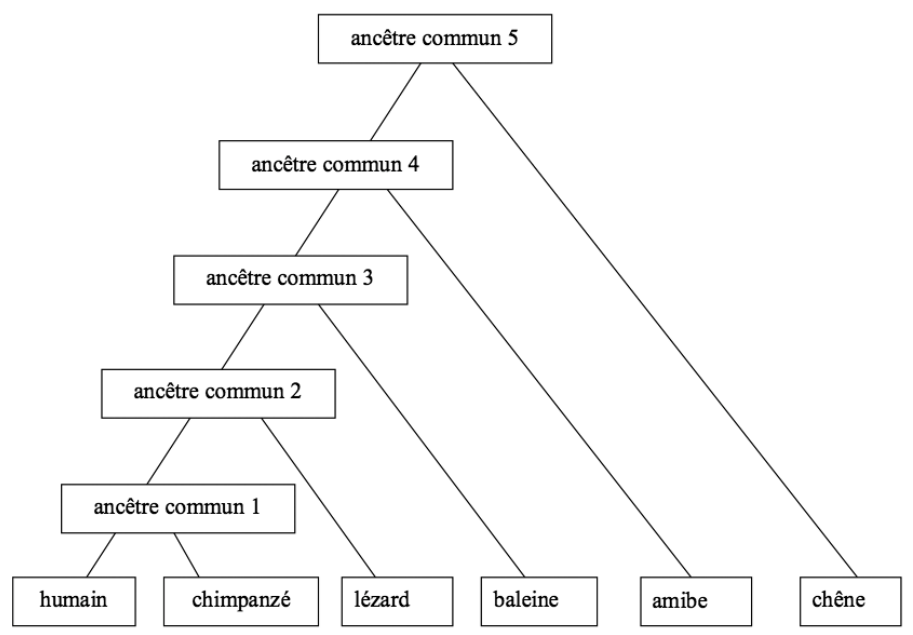


Certes, ce type de schéma paraît, au premier abord, nettement moins linéaire et scalaire que le précédent, puisqu'il dessine un arbre, avec des embranchements qui représentent des «ancêtres communs ». Rigoureusement parlant, il n'est pas incompatible avec une interprétation darwinienne. Toutefois, si l'on y prend garde, on s'aperçoit que l'échelle des êtres régule ici encore profondément ce dispositif graphique, tel qu’il est interprété par celui qui l'a tracé.

Tout d'abord, les noms des espèces sont disposés le long d'une ligne qui reproduit manifestement une échelle des êtres globalement graduée, semble-t-il, selon un critère anthropocentré, hiérarchique, qui renvoie au degré de proximité avec l'être humain: à une extrémité, le chêne et l'amibe, à l'autre, l'homme, et juste avant ce dernier, le chimpanzé. Or on peut fort bien dessiner un arbre darwinien sans que l'homme ou l'amibe se trouvent à une extrémité, comme c'est le cas dans le schéma suivant (Schéma 3), l’un des très rares qui soient compatibles sans ambiguité ${ }^{8}$ avec ce que dit la biologie actuelle à propos de l'évolution? :

Schéma 3.

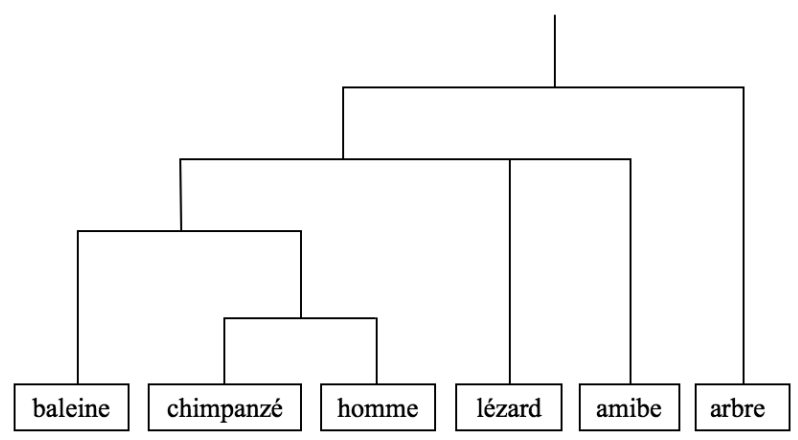

8 II comporte toutefois une erreur, mais qui est simplement factuelle, à propos du lien entre le lézard et l'amibe.

9 Du reste, les manuels scolaires français privilégient aujourd'hui ce type de représentation, précisément pour manifester avec force la rupture profonde qu'opère le darwinisme avec l'échelle des êtres et l'anthropocentrisme (voir Lecointre, 2009). 
Ensuite, le mode opératoire suivi par les étudiants qui ont tracé des dessins semblables au schéma 2 atteste que l'échelle des êtres est bien au principe de la représentation des espèces qu'ils donnent à voir. En effet, comme le suggère la simple vue de ces schémas, et comme l'a confirmé l'observation de quelques étudiants au moment où ils les ont réalisés, le procédé suivi pour les bâtir a consisté à commencer par écrire sur une ligne le nom des différentes espèces en les distribuant le long d'une échelle anthropocentrée; puis, dans un second temps, à dessiner les embranchements - ou, si l'on veut, les chevrons - censés les relier deux à deux. Dans ces schémas, les embranchements sont donc engendrés à partir de cette échelle. Par conséquent, la référence à des ancêtres communs est pensée sur fond d'une conception scalaire et anthropocentrée du vivant : l'arbre cache ici une échelle.

Au total, on ne trouve que 4 schémas dans lesquels le schème de l'échelle des êtres anthropocentrée ne paraît jouer aucun rôle organisateur, que ce soit explicitement, par linéarisation directe des relations tracées, ou implicitement, par inscription dans un arbre (Tableau 4).

Tableau 2. Prégnance de l'échelle des êtres anthropocentrée (en \%)

\begin{tabular}{|c|c|c|c|c|}
\hline \multicolumn{2}{|c|}{ SCHÉMAS AVEC ÉCHELLE DES ÊTRES } & $\begin{array}{c}\text { SCHÉMAS } \\
\text { SANS ÉCHELLE } \\
\text { DES ÊTRES }\end{array}$ & TOTAL \\
\hline Par linéarisation des liens & $\begin{array}{c}\text { Inscription de l'échelle } \\
\text { des êtres dans les arbres }\end{array}$ & autres & & \\
\hline 72 & 20 & 4 & 4 & 100 \\
\hline & 96 & 4 & \\
\hline
\end{tabular}

En résumé, l'écrasante majorité de ces schémas s'organise donc à la fois autour d'un schème transformiste et d'un schème scalaire anthropocentré (Tableau 2). La conception de la transformation des espèces - ou, si l'on veut, de l'évolution - qui se donne ici à voir se ramène donc à une progression temporelle linéaire, orientée de toute nécessité vers l'apparition de l’homme. Une forme de téléologie, de progrès, s’y laisse lire, qui ne laisse guère de place au hasard. 
On remarquera que cette représentation de l'évolution est fort répandue en France dans l'espace public - dans les médias comme dans la vulgarisation scientifique (Guillo, 20I5). En effet, l'«évolution» y est fréquemment présentée comme un processus linéaire et ascendant de complexification du vivant, qui commence avec des êtres à l'organisation fruste et sommaire, dépourvus d'esprit, et qui se poursuit à travers les âges pour aboutir à l'être humain, doté des facultés mentales les plus élaborées. Dans un tel cadre, les animaux, actuels et passés, apparaissent comme les simples étapes d'un processus qui a conduit, suivant une implacable nécessité, à l'avènement de l'humanité, espèce qui surplombe par l'éminence de ses capacités le règne vivant. Cette représentation est parfaitement résumée par la formule très répandue: «l'homme descend du singe».

Or une telle représentation est fort éloignée de la manière dont la biologie actuelle conçoit l'évolution (voir, par exemple, Mayr, 200I ; Dawkins, 2009). Dans la biologie contemporaine, l'homme ne «descend» pas «du singe». L’un et l'autre sont cousins. Plus précisément, les humains actuels et les singes actuels ont un ancêtre commun, qui a des caractéristiques et un nom propres. L’homme n'est plus au sommet de l'évolution. Il n'est qu'une petite branche parmi une infinité d’autres de l’arbre généalogique immense que composent les êtres vivants. La complexité de son cerveau est une particularité, et non une supériorité. Elle en fait un être adapté à certains environnements, mais pas nécessairement à d'autres. L'évolution n’a donc pas de direction. Elle ne dessine aucun progrès. Elle est largement livrée au hasard des vicissitudes écologiques dans lesquelles vivent les populations concrètes d’êtres vivants. En résumé, comme on l'a évoqué plus haut, la conception scientifique actuelle de l'évolution rompt radicalement avec les cinq schèmes suivants : la conception fixiste des espèces, l'échelle des êtres anthropocentrée, la négation du rôle du hasard, la conception essentialiste des espèce ${ }^{10}$ et la téléologie. Or on retrouve quatre de ces schèmes dans la conception de l'évolution comme complexification progressive et croissante des êtres vivants (voir Guillo, 2015) : seul le schème du fixisme est absent, remplacé par une conception transformiste des espèces. 
En d'autres termes, les étudiants que nous avons interrogés affichent globalement, certes, une adhésion à ce que dit la science et à «l'idée d'évolution ». Mais cette adhésion revendiquée recouvre en réalité une sorte de malentendu. Car la représentation qu'ils associent ici au mot «évolution » est fortement marquée par les schèmes évoqués précédemment, avec lesquels Darwin et la biologie actuelle (voir Mayr, 200I) ont profondément rompu. En définitive, cet «évolutionnisme » affiché par les acteurs est donc très différent de ce que dit la science aujourd'hui, bien que les acteurs eux-mêmes pensent avoir des convictions qui en sont fort proches.

\section{I.3. UN ÉVOLUTIONNISME PROCHE DE CERTAINES FORMES DE CRÉATIONNISME}

Un tel constat conduit à formuler deux remarques. Tout d'abord, on comprend pourquoi la conception de l'évolution qui a cours dans le grand public français peut s'accompagner, parfois, sans peine, d'une forme d'adhésion à la religion, en raison de ses fortes résonnances téléologiques, essentialistes et anthropocentriques. Par exemple, dans le courrier de Sciences \& Vie que nous avons analysé (Guillo, 2015), beaucoup de lettres insistent sur l'absence de contradiction entre la foi religieuse - du moins la croyance en l'existence d'une entité métaphysique supérieure -, d'une part, et l'adhésion à l'idée d'«évolution » du vivant, ou au «darwinisme », d'autre part. Plus encore, aux yeux de beaucoup de ces lecteurs, l'évolution, telle qu'ils la comprennent, requiert une forme d'explication religieuse, du moins métaphysique. Car dans un tel cadre, il faut bien expliquer le sens qu'elle semble avoir, rendre compte de la main providentielle qui semble la diriger vers une forme de progrès ordonné et anthropocentré. Et même lorsque le hasard est invoqué, il a tous les attributs d'une cause finale transcendante, dont l'action est quelque peu mystérieuse. Finalement, le contenu et la structure de ces partis pris attestent, ici encore, qu'il n'y a pas de lien indéfectible, ou nécessaire, entre l’adhésion à la religion, d'une part, et le refus de l'idée d'évolution, le rejet de la science, ou l’adhésion affichée au créationnisme, d’autre part. 
En outre, dans la mesure où elle renferme quatre des schèmes créationnistes évoquées plus haut, cette conception de l'évolution répandue en France n'est pas aussi éloignée qu'on pourrait le croire de celle de certains «créationnistes » affichés. Peut-être est-il même possible d’aller plus loin. En effet, une partie des créationnistes actuels, notamment les représentants du courant influent de l'Intelligent design, admettent une forme de transformisme. Si bien que leurs croyances sur l'origine et le devenir des espèces vivantes sont finalement, en substance, presque identiques à celles de nombre d'individus qui, en France, disent adhérer à «l'évolution», au «darwinisme» et être hostiles $\mathrm{au}$ «créationnisme». Les représentations de la nature affichées respectivement par les premiers et les seconds ont presque le même contenu, bien qu'elles soient mutuellement perçues par les acteurs comme opposées. Et elles sont tout aussi éloignées, l'une et l'autre, de ce que dit aujourd'hui la science sur cette question, même si les tenants de l'«évolutionnisme » sont considérés et se considèrent comme étant plus proches du discours scientifique.

Bien entendu, il n'est pas question de contester qu'il existe une différence capitale entre les postures de ces deux catégories d'acteurs: les tenants de «l'évolutionnisme» dans le grand public français se réfèrent à la biologie de l'évolution et, plus largement, à la méthode scientifique, et considèrent que les faits doivent trancher à propos des phénomènes naturels; les créationnistes militants considèrent, quant à eux, que la religion et les autorités qui la représentent doivent avoir le dernier mot. En somme, on trouve ici deux positionnements qui peuvent être radicalement différents à propos de la vérité, de la manière de concevoir les arguments qui l'étayent et de la place du discours de la science dans la société. Il reste que, si l'on s'en tient strictement à la représentation de la nature vivante, les conceptions en présence sont beaucoup plus proches que ne le pensent les acteurs eux-mêmes. En définitive, les différences réelles entre les uns et les autres résident bien davantage dans un registre ontologique et épistémologique - le rapport à la vérité - et social - la place de l'institution scientifique -, que dans le contraste effectif entre leurs représentations de la nature, autrement dit dans un registre relevant de la conception positive du monde naturel. C'est pourquoi on ne peut rendre compte de la logique qui commande les croyances en présence, leurs perceptions croisées et les conflits 
qui les entourent, en soutenant que ce jeu complexe trouve l'une de ses sources essentielles dans la contradiction entre deux représentations du vivant, l'une défendue par la science de l'évolution, l’autre par la religion.

\subsection{DES REPRÉSENTATIONS FLOUES ET UNE INDIFFÉRENCE RELATIVE À L'ÉGARD DE LA QUESTION}

Dernier fait observé sur ces terrains - fait fort important, sur lequel nous reviendrons: une série d'entretiens menés autour du questionnaire avec les étudiants interrogés et, plus largement, avec des non-spécialistes en biologie, montrent qu'en réalité beaucoup d’acteurs n’ont pas un avis extrêmement précis et défini sur cette question qui, parfois, les intéresse relativement peu dans le détail. Pour le montrer plus spécifiquement, nous avons posé à des professeurs des écoles un problème semblable à ceux qui servent de point d'appui à de nombreuses enquêtes sur la réception et la mécompréhension du darwinisme. Dans ces expériences, d'ordinaire, le sociologue ou l'anthropologue, au terme de l'entretien, met en ordre les phrases prononcées par les individus de manière à obtenir un système de propositions qui est censé exprimer leurs croyances sur la question posée (voir, par exemple, Evans, 2000; et, pour la France, Bronner, 2007 et 20I4). Dans l'expérience que nous avons menée, nous avons enregistré les dialogues entre l'expérimentateur et la personne interrogée, afin de faire ressortir les modalités d'expression, les propriétés et l'intensité de la conviction qui s'exprime au fil des réponses fournies. Le problème que nous avons posé portait sur l'évolution du cou des girafes ${ }^{11}$ :

E - Comment expliqueriez-vous à un enfant les étapes de l'évolution qui ont mené à la formation du long cou des girafes actuelles, sachant que les ancêtres des girafes avaient des cous en fait courts?

JD - J'écris ça?

E - Non non tu expliques.

JD - Je parle là.

II E désigne l'expérimentateur. JD sont des initiales fictives pour la personne interviewée. Pour le détail de cette expérience, voir Guillo, 2015. 
E - Oui, oui tu parles.

JD - Alors elles avaient des cous courts.

E - L'ancêtre des girafes, il a un cou très court, et comment est-ce qu'on est arrivé à la première girafe avec un grand cou ?

JD - Bah c'est par rapport à l'alimentation parce que ils ont dû...

E - Oui. (Rire). Je ne sais pas moi, ne me regarde pas comme ça moi j'en sais rien.

JD - Faut bien que je parle à quelqu’un.

E - Oui, oui, vas-y.

JD - Et pourquoi est-ce qu'ils ont dû... Ça veut dire qu'il n'y avait plus à manger par terre ou heu...

E - Ouais.

JD - Pourquoi il n'y avait plus à manger par terre? Bah y'a eu des évolutions climatiques qui ont fait qu'il n'y avait plus à manger par terre mais en même temps ça veut dire qu'il y a eu une sécheresse donc normalement il ne devrait plus y avoir d'arbres non plus... Donc pourquoi... Pour avoir accès à de la nourriture qui n'était pas mangée par d'autres animaux. Je ne sais pas, j’ai pas la réponse.

E - D’accord. Donc un changement dans l'environnement, enfin dans la végétation.

JD - Adaptation au fait que... Grâce au long cou elles ont accès à des branches auxquelles n'ont pas forcément accès tous les animaux qui mangent les végétaux.

E - D'accord, et comment ça s'est fait? Comment il y a eu la première qui avait un grand cou au milieu des girafes à petit cou? Comment ça s'est fait?

JD - Bah c'est une évolution... Ben... génétique quoi, progressive.

E - D’accord.

JD - Naturelle, qui fait appel à l'adaptation quoi en fait. Elles n'ont pas eu le choix en fait, soit... Enfin il y a eu un moment... Pour que l'espèce prospère et survive, il a fallu qu'elle s'adapte et elle a réussi à s'adapter quoi. 
E - D’accord.

JD - Donc avec un cou long, ben ça, ça le faisait plus pour s'alimenter quoi.

E - Ok. C'est bon?

JD - Oui.

Il pourrait être tentant de considérer, à la lecture de cet extrait, que JD mobilise dans ses réponses un schème téléologique - «ça le faisait plus pour s'alimenter ». En poussant un peu l'entretien, il n’aurait guère été difficile d'obtenir de cette personne des phrases susceptibles d'être interprétées comme des manifestations d'une conception de l'évolution de type «lamarckien ». Telles sont d'ailleurs les conclusions auxquelles parviennent beaucoup de recherches sur cette question (Bishop et Anderson, 1990 ; Evans, 2000 ; et, pour la France, Bronner, 2007 et 2014).

Toutefois, une telle interprétation des données sous-estime toute la distance qui sépare des mots ou des phrases aux accents finalistes - que prononcent effectivement JD ici -, d'une part, et des croyances finalistes, au sens fort, d'autre part, c'est-à-dire des sortes de théories - en la circonstance, de type lamarckien ou, si l'on veut, téléologiques. En effet, si l'on s'attache aux éléments du dialogue qui traduisent le rapport de l'individu à la croyance qu’on lui prête, il apparaît que les quelques mots qu'il prononce - «c'est une évolution», «qui fait appel à une adaptation» - semblent très rapidement constituer à ses yeux une explication suffisante, qui répond à la question avec un degré de précision satisfaisant. Or ces mots, dans l'esprit de l'individu, ont un sens manifestement vague. Ils ne paraissent pas renvoyer à un champ de questionnements qu'il aurait l'habitude d'explorer en profondeur, à propos duquel il aurait un avis, des convictions définies. Certes, un contenu cognitif affleure ici: la représentation qui se déploie n’est pas vide de sens. Mais il serait bien hasardeux de considérer qu'il peut être traduit sous forme de propositions dotées d'un contenu précis, autrement dit sous la forme d'une théorie. Une telle interprétation serait d'autant plus contestable que, pour obtenir une réponse, l'expérimentateur a dû chaque fois pousser 
l'individu à fournir des explications plus précises, que ce dernier n'a pas données spontanément. Plus largement, dans beaucoup d'entretiens que nous avons menés, les échanges ont été jalonnés de manifestations de gêne, de rires, d’aveux d’ignorance - «j'en sais rien » - ou de délégation de la réponse à des autorités scientifiques. Autant de signes qui traduisent un engagement d'une faible intensité, une forme de distance et d'indifférence à l'égard de la thématique abordée. Or ces différentes manifestations doivent impérativement être prises en compte dans la description que le chercheur donne de telles croyances. Elles doivent le conduire à caractériser celles-ci comme des représentations intrinsèquement floues pour une part, relativement indéfinies dans leur contenu. Cette propriété - ou cette variable - est très rarement évoquée dans les enquêtes sur les croyances relatives à l'origine des espèces. Pourtant, elle joue assurément un rôle capital dans l'articulation entre le discours scientifique, la foi religieuse et les représentations ordinaires des objets naturels, comme on tentera de le développer dans la troisième partie.

\section{LE MAROC:}

\section{QUEL CRÉATIONNISME TROUVE-T-ON?}

Ces investigations ont été poursuivies au Maroc dans le but de procéder à des comparaisons avec une situation a priori fort différente de celle que l'on observe en France. La religion occupe en effet au Maroc une place majeure: l'islam est religion d'État. Or, comme la Bible, le Coran recèle des passages dans lesquels la création de l'homme par Dieu est explicitement évoquée. Un tel contexte est par conséquent très instructif pour tester l'incidence d'une religion fortement implantée dans l'espace public - en l'occurrence, ici, l'islam - sur la représentation de l'origine des espèces. 


\section{I. LE PROBLÈME GÉNÉALOGIQUE SOUMIS À DES ÉTUDIANTS MAROCAINS}

Pour effectuer une première comparaison avec le cas français, nous avons soumis le problème portant sur la généalogie des êtres vivants aux étudiants marocains inscrits en première et en deuxième année d'une école de science politique $^{12}(\mathrm{~N}=97)$. Ces étudiants sont pour la plupart issus de milieux aisés. Ils ont suivi un enseignement de biologie au cours de leurs études en lycée, soit dans les établissements français implantés au Maroc - une bonne partie des élites marocaines suit cette filière - soit dans le système d'enseignement marocain privé ou - beaucoup plus rarement - public. Les étudiants de notre échantillon ont très majoritairement suivi la seconde filière - celle de l'enseignement marocain privé.

Les établissements d'enseignement français au Maroc suivent les mêmes programmes que leurs homologues implantés en France. Les manuels de biologie de ces lycées sont donc ceux des lycées français et le darwinisme y est enseigné dans les filières scientifiques. En revanche, dans les manuels de l'enseignement marocain, la question de l'origine des espèces est esquivée. La théorie de l'évolution et le darwinisme ne sont pas évoqués, ni pour être critiqués, ni pour être exposés, ou défendus contre le créationnisme. Et l'on n’y trouve pas davantage d'évocation explicite ou implicite de quelque création divine des êtres vivants. Ces manuels sont pourtant fort riches, complets et détaillés en information dans beaucoup de domaines des sciences de la vie et de la terre, comme la génétique, la biochimie, l'écologie ou encore la géologie. Dans ces parties du programme, ils reprennent généralement, en

12 Nous avons choisi une population d'étudiants en science politique, et non en science - comme en France - pour la raison suivante. Notre objectif, dans cet article, est de montrer que les représentations des acteurs ordinaires sur ce thème ne sont pas exactement celles que l'on pourrait attendre compte tenu des contextes publics respectifs dans lesquels ils sont plongés dans ces deux pays - pour le dire vite, lä̈que, pour la France, marqué par la religion, pour le Maroc. II nous a donc semblé que notre démonstration aurait plus de force si l'on parvenait à montrer, d'un côté, qu'en France, même chez les étudiants qui ont reçu une solide formation scientifique, la représentation est assez éloignée de ce que dit la science, et que, d'un autre côté, au Maroc, même chez les étudiants qui suivent des cursus éloignés de la science, les représentations sont à la fois moins hostiles au darwinisme et moins directement commandées par la foi qu'on ne pourrait le penser. 
arabe, les lignes directrices pédagogiques des manuels français. Toutefois, la thématique de l'origine des espèces, ses implications - en matière de classification, ou encore de génétique - et les débats qu’elle suscite sont, quant à eux, ignorés et passés sous silence. Cette manière d'esquiver la question, ainsi que les controverses qu'elle entraîne dans son sillage, est assez symptomatique du mode de présence très particulier qui semble être celui du darwinisme dans l'espace public marocain. Il est enseigné dans les lycées français. Il n’est pas officiellement contesté, ne donne pas lieu à de vives polémiques publiques, du moins à ce jour, notamment dans les médias. Mais dans le même temps, le créationnisme paraît très largement répandu dans les diverses couches de la population, fortement relayé par les autorités religieuses.

\subsection{LES MODIFICATIONS APPORTÉES AU PROTOCOLE EXPÉRIMENTAL DANS CE CONTEXTE}

Le questionnaire a été adapté aux spécificités de ce contexte. Tout d’abord, la question, ainsi que le nom des êtres vivants, ont été traduits en arabe dialectal marocain. Nous avons également remplacé les mots «amibe» et «chêne » par les mots «bactérie » et «arbre» sous les photographies correspondantes, les personnes interrogées paraissant mieux identifier les seconds que les premiers dans les essais que nous avons effectués pour tester le questionnaire. Par ailleurs, comme nous pouvions supposer, a priori, qu'il y aurait bien davantage de schémas de type créationniste qu'en France, nous avons choisi de proposer trois réponses possibles aux étudiants, afin de mieux analyser le contenu de leurs convictions. Ces trois réponses, formulées en arabe dialectal, sont les suivantes :

1. «il n’existe aucun lien généalogique entre ces êtres ».

2. «il existe des liens généalogiques entre certains de ces êtres».

3. «il existe des liens généalogiques entre tous ces êtres».

Pour les réponses 2 et 3, l'on demandait de tracer un arbre généalogique. Un espace était laissé libre pour d'éventuels commentaires. 
La distinction entre la réponse 2 - il existe un lien généalogique entre certains des êtres vivants mentionnés ici - et la réponse 3 - il existe un lien généalogique entre tous ces êtres vivants - avait pour objectif de permettre aux individus d'établir certains liens, et d'en écarter d'autres. À travers cette distinction, nous voulions tester l'hypothèse selon laquelle les réticences visà-vis de l'idée d'évolution trouvent leur source dans le caractère sacré accordé par la religion à l'être humain, conformément à la thèse de la sécularisation et plus spécifiquement à celle de Freud. Si cette hypothèse était exacte, l'on devrait observer que les individus consentent d’autant plus volontiers à établir des liens généalogiques entre les êtres vivants, que ceux-ci paraissent éloignés de l'être humain. Corrélativement, le lien qu'ils devraient le plus répugner à établir est l'ascendance entre l'être humain et le singe, ce lien apparaissant, a priori, comme le plus sacrilège au regard de la religion ${ }^{13}$.

Tels sont donc, au total, les aménagements que nous avons apportés à ce problème sur le terrain marocain. Les résultats obtenus sont différents, sous certains aspects, de ceux que nous avons recueillis auprès des étudiants français. Pourtant, comme les précédents, ils attestent que le tableau des croyances collectives, sur ce thème, est fort éloigné de ce que suggère l'hypothèse selon laquelle le refus du darwinisme serait simplement le produit d'un relâchement cognitif, causé par une foi aveugle dans la religion, ou accompagnant celle-ci.

\subsection{LA PRÉSENCE MINORITAIRE DU FIXISME GÉNÉRALISÉ}

Premier constat important, le fixisme généralisé à toutes les espèces - autrement dit l'hypothèse d'une absence totale de lien généalogique entre les êtres vivants (réponse I) - n'est pas majoritaire dans l'ensemble des réponses fournies. Il est affiché par 36,I \% des étudiants interrogés, contre 29,9\% pour le transformisme limité (réponse 2) et I8,6\% pour le transformisme généralisé (réponse 3), soit au total $48,5 \%$ pour les deux variantes de transformisme (Tableau 3).

13 Comme en France, le questionnaire était anonyme. II a été distribué aux étudiants à la suite d'un examen, sans explication particulière sur le thème abordé (sur les raisons de cette absence d'explication, voir infra). Ce dispositif évitait le face-à-face avec un expérimentateur, situation qui entraîne des biais considérables sur de semblables questions. Cela évitait également qu'ils communiquent entre eux, avant, après ou pendant qu'ils répondaient à ce questionnaire. 
Le créationnisme de type fixiste est donc bien présent dans cette population étudiante issue de milieux aisée, mais il est loin de faire l'unanimité ${ }^{14}$.

Tableau 3. Fixisme ou transformisme

\begin{tabular}{|l|c|c|c|c|c|}
\hline & $\begin{array}{c}\text { RÉPONSE I: } \\
\text { ABSENCE DE LIEN }\end{array}$ & $\begin{array}{c}\text { RÉPONSE 2: } \\
\text { LIENS ENTRE CER- } \\
\text { TAINES ESPĖCES }\end{array}$ & $\begin{array}{c}\text { RÉPONSE 3: } \\
\text { LIENS ENTRE } \\
\text { TOUTES LES } \\
\text { ESPĖCES }\end{array}$ & NON-RÉPONSES & TOTAL \\
\hline $\begin{array}{l}\text { VALEUR } \\
\text { ABSOLUE }\end{array}$ & 35 & 29 & 18 & 15 & 97 \\
\hline$\%$ & 36,1 & 29,9 & 18,6 & 15,4 & 100 \\
\hline
\end{tabular}

\subsection{UN TRANSFORMISME ASSEZ PROCHE DE CELUI DES ÉTUDIANTS FRANÇAIS}

L’analyse détaillée des schémas proposés par les étudiants qui ont choisi une réponse transformiste - partielle (2) ou généralisée (3) - est fort riche en enseignements supplémentaires. Tout d'abord, beaucoup de schémas n'établissent de lien qu'entre certains êtres vivants - réponse 2, soit 29,9\% du total -, laissant les autres de côté, ou ne les mentionnant pas, à l'exemple du schéma suivant (Schéma 4).

14 On notera également que la grande majorité des étudiants qui ont refusé d'établir un lien généalogique quelconque - réponse I - n'ont pas formulé de remarques manifestant avec ostentation et véhémence leur attachement à la foi dans l'espace proposé sur la feuille de réponse pour des commentaires éventuels. Seuls 3 étudiants ont évoqué Dieu ou la religion dans cet espace. 
Schéma 4.

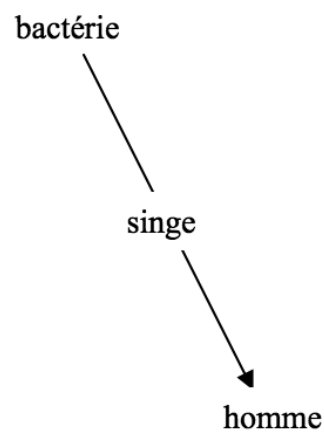

Note: rédigé en arabe dialectal par l'étudiant.

Plus généralement, on ne trouve dans cet ensemble aucun schéma généalogique qui puisse être considéré comme étant rigoureusement conforme à la théorie de l'évolution ${ }^{15}$. Certes, quelques schémas présentent une forme arborescente, l'un d’entre eux faisant même référence à des «ancêtres communs» (Schéma 5).

Schéma 5.

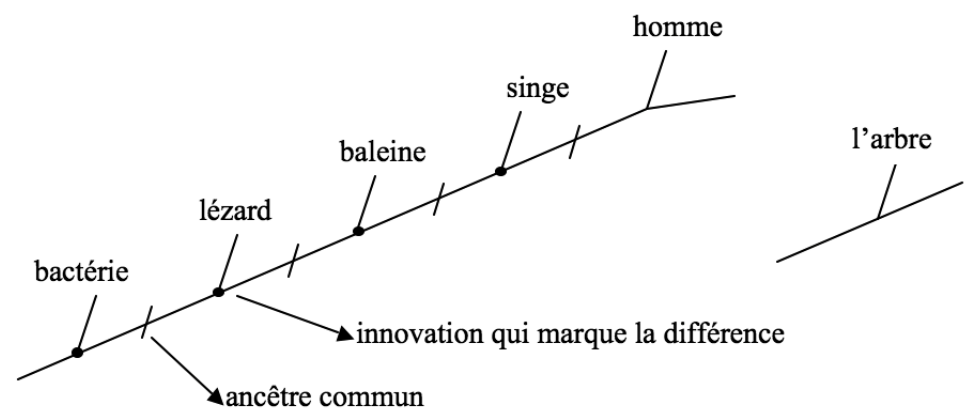

Note: rédigé en français par l'étudiant.

I5 De tels schémas étaient cependant également fort rares - au nombre de 4 -, rappelons-le, chez les étudiants français. 
Toutefois, la plupart des schémas recueillis sont composés de lignes reliant directement les êtres deux à deux, et beaucoup plus rarement d'embranchements susceptibles de figurer de tels ancêtres. L'être humain et le singe, en particulier, ont été reliés presque immanquablement par une telle ligne, conformément à la formule : «l'homme descend du singe ». Et l'homme a été situé chaque fois à une place manifestement particulière, éminente, soit à une extrémité du schéma, soit à part.

En définitive, on retrouve chez ceux qui ont manifesté une forme d'adhésion au transformisme - limité ou général - les mêmes schèmes que dans la représentation «évolutionniste » de la nature observée en France : un transformisme habité par l'échelle des êtres anthropocentrée, qui implique une forme de téléologie et une négation du rôle du hasard.

\subsection{LE RÔLE MAJEUR D'UN CRITÈRE POSITIF: LES DIFFÉRENCES D'APPARENCE ENTRE LES ÊTRES VIVANTS}

Un fait sans doute plus important encore se dessine dans ces schémas transformistes. Ce fait se rapporte à l'hypothèse que nous voulions tester en distinguant les réponses 2 et 3 . Ces schémas suggèrent que le refus d'établir un lien généalogique entre les êtres vivants n’a manifestement pas toujours - ou pas uniquement - procédé d'un attachement aux exigences de la foi, contre celles de la raison. Comme on l'a souligné plus haut, si la foi était le seul principe guidant le choix de relier, ou non, chaque être à un autre, l'on devrait observer dans l'ensemble des liens établis une sous-représentation du lien humain/singe, celui-ci étant lesté de la plus forte charge sacrilège. Or l'on observe précisément l'inverse : contrairement à ce que prédit cette hypothèse, la relation humain/singe est, de loin, celle qui a été privilégiée par les étudiants qui ont tracé des schémas généalogiques. Ainsi 68, I \% de ces étudiants - soit $33 \%$ de l'ensemble des étudiants interrogés - ont établi un lien entre ces deux êtres vivants. Viennent ensuite, assez loin derrière, à égalité, les relations humain/bactérie et chêne/bactérie, établies par 51,I \% de ces étudiants, c'est-à-dire 24,7\% de l'ensemble (Tableau 4). 
Tableau 4. Nombre de liens directs établis deux à deux entre espèces

\begin{tabular}{|c|c|c|c|c|c|c|c|c|c|c|c|c|c|c|c|}
\hline & 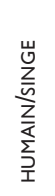 & 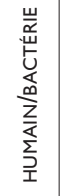 & 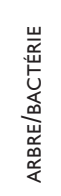 & 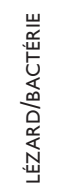 & 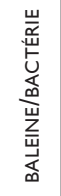 & 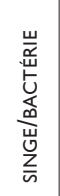 & 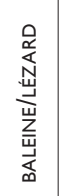 & 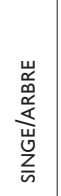 & 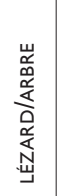 & 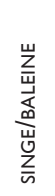 & 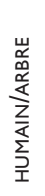 & 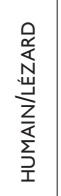 & 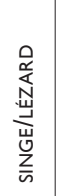 & 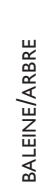 & 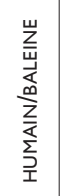 \\
\hline VALEUR ABSOLUE & 32 & 24 & 24 & 23 & 22 & 21 & 14 & 11 & 10 & 9 & 8 & 6 & 6 & 6 & 4 \\
\hline $\begin{array}{l}\% \text { PAR RAPPORT } \\
\text { AUX RÉPONSES } \\
2 /+3 /\end{array}$ & 68,1 & 51,1 & 51,1 & 48,9 & 46,8 & 44,7 & 29,8 & 23,4 & 21,3 & 19,1 & 17 & 12,8 & 12,8 & 12,8 & 8 \\
\hline $\begin{array}{l}\% \text { PAR RAPPORT } \\
\text { AU TOTAL DES } \\
\text { RÉPONSES }\end{array}$ & 33 & 24,7 & 24,7 & 23,7 & 22,7 & 21,7 & $\mid 4,4$ & 11,3 & 10,3 & 9,3 & 8,2 & 6,2 & 6,2 & 6,2 & 4 \\
\hline
\end{tabular}

Par ailleurs, l'être vivant le plus fréquemment relié à un autre - quel qu'il soit - est l'être humain. On le trouve ainsi associé à une autre espèce dans $89,4 \%$ des schémas avec lien, soit $43,3 \%$ du total des questionnaires distribués. Viennent ensuite le singe - 80,9\% (39,2\% du total) - puis la bactérie - 78,7\% (38, I \% du total). L’individu le plus délaissé est l’arbre : 46,8\% (22,7\% du total) (Tableau 5).

Tableau 5. Nombre de liens établis pour chaque espèce avec d'autres espèces

\begin{tabular}{|l|c|c|c|c|c|c|}
\hline & HUMAIN & SINGE & BACTÉRIE & LÉZARD & BALEINE & ARBRE \\
\hline VALEUR ABSOLUE & 42 & 38 & 37 & 29 & 27 & 22 \\
\hline $\begin{array}{l}\text { \% PAR RAPPORT } \\
\text { AUX RÉPONSES } \\
2 /+3 /\end{array}$ & 89,4 & 80,9 & 78,7 & 61,8 & 57,4 & 46,8 \\
\hline $\begin{array}{l}\text { \% PAR RAPPORT } \\
\text { AU TOTAL DES } \\
\text { RÉPONSES }\end{array}$ & 43,3 & 39,2 & 38,1 & 29,9 & 27,8 & 22,7 \\
\hline
\end{tabular}


Ces valeurs indiquent que, parmi les étudiants qui ne souscrivent pas à un fixisme généralisé, l'être humain est de très loin l'espèce avec laquelle sont établis préférentiellement les liens. Plus spécifiquement, ces étudiants paraissent nettement moins répugner à tracer un lien généalogique entre un être humain et un singe, que tout autre lien envisageable dans le problème posé - I5 liens entre deux êtres sont ici possibles (Tableau 4). Le positionnement à l'égard du transformisme ne paraît donc pas motivé ici, fondamentalement, ou uniquement, par la foi. Car si tel était le cas, on devrait constater les plus vives réticences à établir des liens généalogiques lorsque l'être humain est impliqué.

De telles données invitent à formuler une toute autre hypothèse à propos des croyances qui affleurent dans ces schémas. Elles suggèrent qu'un critère d'ordre proprement positif - ou, si l'on veut, rationnel, au sens large - et non simplement d'ordre émotionnel - soutenu par la foi - joue ici un rôle capital dans la formation des convictions relatives à la question du transformisme et du fixisme ${ }^{16}$. Ce critère est le degré de ressemblance perçue entre les êtres vivants. En effet, la hiérarchie que donnent à voir les tableaux 4 et 5 suggère que les individus répugnent d'autant plus à tracer des liens généalogiques entre deux êtres vivants que ceux-ci ont une apparence dissemblable - si l'on excepte toutefois le cas, très particulier ${ }^{17}$, de la bactérie. Il semble donc que les acteurs refusent ici d’admettre l'existence de liens généalogiques parce que, tels qu'ils les comprennent, ils imposent d’accepter une hypothèse qui leur paraît empiriquement douteuse, à savoir que des êtres d'aspects très différents, comme par exemple l'arbre et la baleine, ou le lézard et l'humain, aient pu un jour donner naissance l'un à l'autre, fût-ce progressivement à travers une lignée extrêmement longue.

16 Plus largement, sur les formes de rationalité à l'œuvre dans les croyances apparemment irrationnelles, voir Boudon, 1992.

17 II est vrai que la bactérie présente une apparence très éloignée des autres êtres vivants présentés ici. On pourrait donc s'étonner de la voir figurer en bonne place. On fera toutefois remarquer que les liens établis avec la bactérie dans les schémas où celle-ci a été reliée à d'autres êtres sont davantage des rapports de composition, que des rapports d'engendrement généalogique à proprement parler (pour des précisions, voir infra). 
Cette hypothèse sur le fondement de ces croyances permet d'expliquer pourquoi la relation entre l'homme et le singe a été plébiscitée : ce sont les deux êtres qui manifestent le plus de ressemblances apparentes sur ces photos. Elle permet également de rendre compte de la mise à l'écart de l'arbre : végétal immobile, il paraît fort loin, en apparence, de tous les autres êtres vivants proposés dans le problème. De manière symptomatique, l'un des étudiants a écrit (en français) : «il n’y a aucun lien ni ressemblances entre ces êtres vivants » (c'est nous qui soulignons). Il est donc possible que la thèse transformiste, telle qu'elle paraît ici comprise - à savoir, en simplifiant : «cet être est le descendant en ligne direct de cet autre être » - heurte chez ceux qui n'y adhèrent pas tout autant - et peutêtre chez certains, avant tout - la raison, que la foi. On peut ainsi supposer que, sur ce thème, il n'y a pas de forte tension, dans l'esprit de ces étudiants, entre ce que dit la religion, d'un côté, et ce que suggèrent les constats factuels et le raisonnement ordinaire, de l'autre. Finalement, sur ce plan encore, l'hypothèse de la sécularisation paraît devoir être sérieusement nuancée.

\subsection{UNE HYPOTHÈSE CONFIRMÉE PAR DES ENTRETIENS MENÉS AUTOUR DU PROBLĖME POSÉ}

Pour affermir ces hypothèses, nous avons mené des entretiens autour de ce questionnaire. L'objectif était, plus largement, de cerner avec davantage de précision la manière dont la question était interprétée, afin de mieux saisir la signification des réponses apportées ${ }^{18}$. Trois enseignements essentiels se dégagent de ces séries d’entretiens.

I8 Nous avons mené ces entretiens auprès des étudiants que nous avons interrogés, après coup, et de manière informelle. Ces entretiens ont été menés individuellement. Ils se sont tenus, pour la plupart d'entre eux, dans les jours qui ont suivi la réponse au questionnaire, parfois juste après celui-ci. Nous avons également recueilli des éléments instructifs dans les entretiens que nous avons réalisés autour de ce questionnaire lorsque nous l'avons bâti et testé, avant de le soumettre aux étudiants. Nous menons actuellement une enquête davantage systématique auprès d'un public beaucoup plus large, incluant des milieux populaires. 
Tout d'abord, aux yeux d'une grande majorité des personnes interrogées, ce sont d'abord et avant tout les dissemblances entre les êtres qui font douter de la possibilité d'un lien généalogique, y compris lorsque l'enquêteur suggère qu'il faut imaginer un temps très long et un nombre de générations immense entre les êtres vivants susceptibles d'être reliés.

Deuxièmement, la question ne semble pas comprise tout à fait de la même manière qu'en France. Ceci tient principalement au fait que les mots «lien» et «généalogie», notamment, peuvent être difficilement traduits en arabe dialectal, comme dans toute autre langue, de manière à recouvrir aux yeux des acteurs d'emblée exactement le même champ sémantique qu'en français. Ainsi, dans la vingtaine de tests préalables que nous avons effectués pour mettre au point ce questionnaire, il s'est avéré que les différents mots susceptibles d'être utilisés pour indiquer aux personnes interrogées que la question posée portait sur l’arbre généalogique dessiné par les liens biologiques, et seulement eux, étaient immanquablement investis d'une signification quelque peu flottante et qui, toujours, implicitement, et même parfois tout à fait explicitement, incluait des liens non biologiques - des liens de filiation par adoption, par exemple -, excluait des liens biologiques - en ne retenant, par exemple, que les liens biologiques patrilinéaires -, dessinait des distances dans la filiation qui ne correspondaient pas exactement aux distances dans la proximité biologique, en raison de distinctions entre les cousins, par exemple, ou même parfois, pour certains mots, renfermait également des liens de parenté analogique et symbolique, parfois même de composition organique ou de cohabitation écologique. Ces deux dernières significations apparaissent d’ailleurs très clairement dans les schémas que nous avons recueillis. En effet, une part non négligeable des étudiants que nous avons interrogés - environ I5\% - ont manifestement interprété cette question en termes compositionnels/génétiques (Schéma 6) - les êtres vivants sont composés d'êtres élémentaires simples - ou écologiques (Schéma 7) - l'arbre donne de l'oxygène aux autres créatures ou le singe se nourrit des fruits de l'arbre, par exemple - plutôt que généalogiques, au sens biologique. 
Schéma 6.

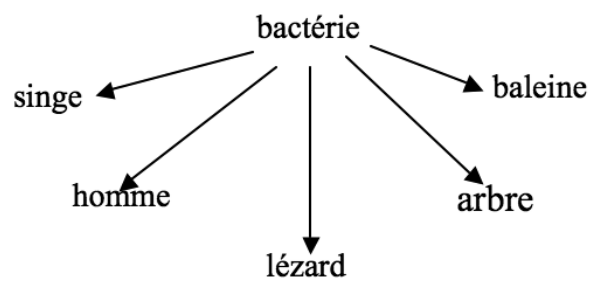

Note: rédigé en arabe dialectal par l'étudiant.

Schéma 7.

«L'arbre est la source de vie de toutes ces créatures parce qu'il leur donne l'oxygène»

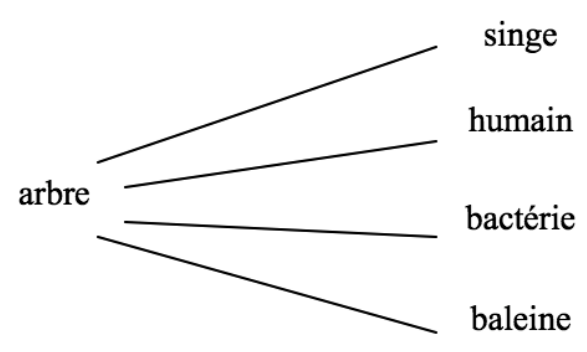

Note: rédigé en arabe dialectal par l'étudiant, y compris la citation.

Au terme de ces tests, l'expression rwābt āl naseb (روابط النسب) nous a paru celle qui dessinait spontanément l'ensemble de relations qui recoupait le mieux - ou le moins mal -, dans l'usage qui semblait être le sien auprès des personnes que nous avons interrogées, l'ensemble dessiné par les liens strictement biologiques, à défaut de le recouvrir exactement.

Bien entendu - et c'est là un point qu'il est essentiel de souligner avec force -, l'idée d'une conception purement biologique de la généalogie est en elle-même parfaitement compréhensible et comprise par ces acteurs, tout comme les groupes d'individus qu'elle dessine et la nature des liens qu'elle tisse entre eux. Simplement, ces groupes et ces liens ne correspondent pas à une catégorie fermement constituée linguistiquement et fréquemment utilisée dans la vie 
courante, laquelle mobilise, à ce jour et dans les populations que nous avons étudiées, des définitions de la généalogie dans lesquelles la proximité biologique n'est pas le critère premier et unique des partitions. Même s'il peut être explicité ici sans difficulté, le concept de généalogie biologique n’est donc pas associé directement à un mot précis et unique, qui en ancrerait et en stabiliserait l'usage, comme c'est sans doute davantage le cas en France aujourd'hui. Dès lors, les individus sont ici mis en situation de devoir manipuler une classe d'éléments - d'individus - et de relations inhabituelles, construites pour la circonstance, et dont ils n'ont pas le temps - et parfois pas la motivation - d'explorer toutes les conséquences et les ramifications logiques.

Le fait d'entourer le questionnaire d'explications détaillées sur le sens des notions en jeu ne permet pas de contourner cette difficulté, contrairement à ce que l'on pourrait croire au premier abord. Lors de nos tests pilotes, nous avons tenté de soumettre ce problème en formulant de telles explications, avant de laisser la personne interrogée répondre. Cette démarche permet d’obtenir des données incontestablement intéressantes. Toutefois, elle ne doit pas pour autant être considérée comme «plus fiable», «moins biaisée» que celle que nous avons choisie, au motif que le sens de la question serait «plus clair». Tout d'abord, les explications préalables semblent renforcer les effets d'autorité, la personne interrogée se sentant alors davantage évaluée que sollicitée pour donner un avis propre. Ensuite, l'expérience montre qu'il est très difficile d'expliquer ce que signifie le lien généalogique au sens strictement biologique, sans commencer d'une manière ou d'une autre à déployer des connaissances biologiques - des faits, mais aussi des concepts théoriques - que les individus n'avaient pas avant qu'on les questionne et qui servent à un moment ou à un autre de points d'appui à la théorie de l'évolution. Pour le dire autrement, d'un point de vue pratique, où faut-il s'arrêter dans l'explicitation de la question posée, pour être certain de recueillir ce que la personne pense, en propre, et non ce qu'on lui a appris? Enfin et surtout, dans nos tests, de telles explications ont souvent paru jeter le trouble chez les personnes interrogées, plutôt qu'elles n'ont clarifié la question: soulignant le caractère étrange, inhabituel, non évident, de la catégorie de «généalogie» ainsi définie, bien souvent, ces 
explications, soit renforçaient l'idée que, décidément, le darwinisme était une singulière bizarrerie, soit inhibaient la disposition à se prononcer sur une telle interrogation, jugée trop nébuleuse et sans objet.

Un tel constat ne doit pas conduire, toutefois, à douter de la fécondité du protocole d'enquête adopté. Il impose simplement de cerner et de tenir compte du sens que les acteurs donnent aux mots utilisés dans la question, fût-il flottant, pour saisir le sens de la proposition sur laquelle ils donnent effectivement un avis, de la question à laquelle ils répondent effectivement sous ces mots. Ainsi nous a-t-il semblé qu’en France, d'un côté, et au Maroc, de l'autre, une partie des individus interrogés ne répondaient pas exactement à la même question, ne se prononçaient pas sur la même hypothèse, lorsqu'on leur demandait simplement: «existe-t-il un lien généalogique entre l'être humain et le singe?». En simplifiant, on peut dire qu'au Maroc les personnes paraissaient davantage répondre à une question du type : «un singe a-t-il pu un jour ou l'autre enfanter un humain ou un être vivant s'en approchant? ». En France, en revanche, la question semblait davantage comprise de la façon suivante: «sur un nombre immense de générations, d’infimes modifications accumulées peuvent-elles amener à la formation d'un humain à partir d'un singe? ».

Ces différents éléments attirent l'attention sur la complexité de ce type de croyances, et sur les difficultés méthodologiques que l'on rencontre quand on cherche à les analyser. Il invite, en particulier, à regarder avec prudence les résultats des enquêtes, parfois rapides, qui visent à établir si les individus, dans tels pays, aire culturelle ou groupe social «croient à l'évolution» ou «sont créationnistes ». Ce que rejettent les uns, ce à quoi adhèrent les autres et ce que dit la science: ces trois ensembles peuvent renvoyer à des catégories, des entités et des questionnements fort différents, même lorsque les individus les perçoivent comme semblables en raison de l'identité des mots qui servent à les désigner.

Troisièmement, dans ces entretiens réalisés au Maroc, le rejet du darwinisme n’a pas paru s'inscrire dans une posture de rejet de la science en général. Religion et science ont été rarement présentées comme des institutions ou des discours en tension, à la différence de ce que l'on peut observer, par exemple, dans les partis pris affichés par certains activistes néo-évangélistes 
américains $^{19}$. Tout à l'inverse, c'est fréquemment au nom de la science - telle qu'elle est perçue par les acteurs -, tout autant que de la religion, sinon davantage, que le darwinisme a été rejeté, quand il l'a étée ${ }^{2}$.

Surtout, comme en France, l'hypothèse évolutionniste, et plus largement la question de l'origine de l'homme et des espèces vivantes elle-même, n'a pas semblé toujours avoir une puissante force sacrilège. Pour tirer des conclusions certaines sur ce point, il faudrait mener une enquête d'une plus grande ampleur. Toutefois, il semble qu'au Maroc, du moins à ce jour, une telle question - largement déconnectée d'implications pratiques directes, à la différence de celles qui touchent par exemple aux mœurs - ne paraît pas toujours cruciale, voire même digne d'intérêt. Certes, nombre de personnes évoquent, lorsqu'on les interroge, la création divine des espèces vivantes. Toutefois, dès lors que l'on cherche à préciser ce que recouvre cette conviction, en les questionnant par exemple sur les mécanismes et l'ordre suivant lesquels cette création s'est effectuée, la plupart d'entre elles ne semblent guère avoir d'avis défini. Le plus souvent, elles ne manifestent pas un vif intérêt pour ces questions. Durant ces entretiens, l'engagement émotionnel reste généralement d'une assez faible intensité, sauf lorsque l'individu interrogé est habité par une foi intense et militante, associée à un discours tourné contre l'Occident.

Certes, beaucoup de personnes prennent également soin de mentionner qu'elles savent que les Occidentaux pensent que «l'homme descend du singe ». Mais cette conception de l'origine de l'homme est fréquemment présentée comme une opinion différente sur un sujet d'une importance somme toute assez mineure; une opinion, certes, quelque peu étrange, mais qui ne paraît pas choquer outre mesure. Sous cet aspect encore, l'hypothèse de la sécularisation semble donc bien fragile. Il en va de même pour l'hypothèse de Freud: le darwinisme ne paraît pas nécessairement infliger quelque «blessure » narcissique au croyant. Non pas parce que celui-ci y adhérerait bon gré mal gré, parce qu'il serait prêt à faire quelque «concession» à la science. Mais

19 Voir Stavo-Debauge, 2012. Sur l'histoire des liens entre le christianisme et la théorie de l'évolution, voir Lecourt, 2007.

20 Tel est le fait qui ressort également d'une enquête que nous menons actuellement sur la manière dont sont présentés les fossiles dans les musées paléontologiques marocains. 
tout simplement parce que le darwinisme, tel qu'il est compris, est parfois regardé comme une sorte de bizarrerie culturelle sur un sujet qui intéresse peu, et non comme une croyance à forte charge blasphématoire.

\section{INDIFFÉRENCE RELATIVE ET FLOU DES REPRÉSENTATIONS DU VIVANT DANS LE GRAND PUBLIC}

Ce rapport distancié à la croyance, que nous avons également souligné plus haut à propos de la France, est assez souvent négligé en sciences sociales dans les enquêtes sur les liens entre le sens commun, la science et la religion ${ }^{21}$. Pourtant, il est d'une importance capitale pour saisir la logique qui commande ces liens et la dynamique des croyances ordinaires sur de telles questions. Ce qui distingue réellement la représentation de la nature vivante que l'on peut prêter au biologiste de l'évolution, d'une part, et celle que l'on peut prêter au non-spécialiste en biologie, non-croyant ou croyant - du moins lorsque celui-ci n'est pas un créationniste militant ou un amateur éclairé, passionné de science -, d’autre part, ce n'est pas véritablement leurs contenus respectifs. C'est d'abord et avant tout leur degré de précision et la force de l'engagement qui leur est associé. Celle du biologiste est très précise et détaillée, celle des deux autres, du croyant et du non-croyant ordinaires, le plus souvent, beaucoup plus floue.

21 Les travaux d'Albert Piette (voir, par exemple, 2003) font sans doute exception sur ce point. Toutefois, ce que nous voulons souligner ici est, nous semble-t-il, différent de ce qu'il explore avec minutie à travers ses recherches sur la distanciation ou le mode mineur. Nous voulons insister, surtout, sur le caractère sémantiquement flou, relativement indéterminé, des représentations individuelles ordinaires sur ces thématiques. En d'autres termes, l'argument développé dans cet article se situe - et reste - sur un plan cognitif, et non sur le plan phénoménologique, ou phénoménographique, de la présence des individus au monde ou de celle des êtres qui les entourent. Et de ce point de vue, le cas que nous présentons ici n'est qu'un cas particulier. Car il n'y a pas de lien univoque et nécessaire entre la force ou les modalités d'un engagement, d'une part, et le degré de précision de la représentation qui l'accompagne, d'autre part. Des engagements très forts peuvent fort bien s'arrimer à des représentations au contenu flou, indéterminé, comme c'est le cas pour beaucoup d'énoncés convoquant Dieu, par exemple. Pour toutes ces raisons, si les arguments présentés ici peuvent être complémentaires avec certaines vues développées par Albert Piette, ils le sont moins avec les aspects de ses travaux qui se situent sur un plan conceptuel éloigné de celui du cognitivisme au sens large, ou qui s'opposent à lui - notamment la phénoménographie, ou les perspectives sur la présence des êtres - ou encore avec les points d'appui qu'il trouve dans une partie de la philosophie analytique de la religion actuelle, difficilement compatible avec le darwinisme. 
C'est en ce sens que, dans bien des cas, il ne peut exister de contradiction réelle entre la première et la seconde - même dans les cas où les individus les perçoivent mutuellement comme contradictoires. Non pas parce que leurs contenus seraient incommensurables, mais simplement parce qu'elles ont des degrés de précision trop différents pour rendre possible la comparaison; parce que l'une a été cultivée dans le détail, au terme d’un engagement fort, par une personne qui lui consacre tout son temps, tandis que l'autre, souvent marquée par une forme d'indifférence relative, déléguée à des autorités jugées compétentes, est laissée en jachère épistémologique par l’acteur. En ce sens, une représentation floue ne s'oppose pas à une représentation précise, quels que soient leurs contenus respectifs. Pour la même raison, bien souvent, il ne peut exister d’adéquation réelle entre le discours scientifique sur l'évolution, d'une part, et les représentations des non-spécialistes qui affichent une adhésion au darwinisme et à la science, d’autre part.

Telles sont, comme on l'a évoqué plus haut, les propriétés qui apparaissent avec acuité lorsque l'on interroge des non-spécialistes en biologie, croyants ou non-croyants, en France comme au Maroc, sur des questions un peu précises, convergentes et détaillées, au-delà des formules générales comme «l'homme descend du singe». Lorsque l'on engage une conversation en formulant de telles interrogations - par exemple, «quels sont les mécanismes qui font que les espèces évoluent? Pourquoi les girafes ont-elles un long cou? Comment les êtres vivants ont-ils été produits, s'il n'y a pas de transformation des espèces? Pourquoi le singe ressemble-t-il à l'homme? Pourquoi les espèces fossiles n'existent-elles plus aujourd'hui ?», etc. - les personnes interrogées, croyantes ou non, favorables à l'évolution ou non, donnent, certes, un avis. Mais fréquemment - du moins sur les terrains que nous avons étudiés - leur discours se tarit vite. Elles délèguent à des autorités les arguments qui peuvent être produits pour étayer telle ou telle affirmation, disent très volontiers qu'elles ne savent pas, rient parfois, sont embarrassées, détournent la conversation, ou signalent clairement que cela ne les intéresse pas. En d’autres termes, souvent, elles n'ont pas a priori d’idées précises, de convictions ou d'intérêt forts pour la question posée. 
Tel est le fait que les sciences sociales doivent assurément prendre ici au sérieux. Souvent, dans les enquêtes sur les représentations, les croyances ou les savoirs, ces hésitations, gênes, délégations ou indifférences affichées sont considérées comme des obstacles méthodologiques qu'il faut franchir, pour parvenir à «la» croyance ou «la» représentation de l'acteur. L'objectif pour le chercheur devient alors de débusquer quelque savoir, représentation, schème de la pratique latents, ou encore des biais cognitifs ou des modules sous-jacents spécialisés dans le traitement d'un type d'information - ici l'information relative à la catégorisation des espèces vivantes et à l'explication de leur devenir (Medin et Atran, I999; Bronner, 2007). Or on peut s'interroger sur ce que fait le chercheur lorsqu'il poursuit ainsi une «croyance». Jusqu'où faut-il aller? Ne risque-t-il pas, en sommant trop fortement l'acteur de fournir des justifications et des explications à propos de questions qu'il ne s'est peut-être jamais vraiment posées, de recueillir en partie un artefact? Ne faut-il pas finalement considérer ces hésitations, ces gênes ou ce désintérêt affiché, d’abord, comme des données cognitives et anthropologiques, plutôt que comme des obstacles méthodologiques? Car après tout, rien n'exclut que, sur certains sujets, les acteurs ne pensent... pas grand-chose, du moins pas grand-chose de précis. Si l'on consulte notre propre expérience du monde, et l'intérêt très inégal que nous portons à la multitude de choses qui le composent, tout porte à croire qu'il en est bien ainsi. Nous n’avons pas une opinion également précise, un intérêt également soutenu, et un engagement émotionnel aussi fort, pour tous les sujets. Si bien que nos différentes représentations n'ont pas un même degré de complexité et de précision. Pour toutes ces raisons, sur beaucoup de thèmes, les croyances des acteurs ne consistent pas en théories, articulant au moyen d'arguments des propositions au contenu défini, et associées à une force de conviction également intense. Ce sont là des cas limites. Pour le dire autrement, sur beaucoup de thèmes, nos représentations sont floues. Et tel est bien ce que l'on observe chez beaucoup d'acteurs, dans le grand public, à propos de la question de l'apparition et du devenir des espèces vivantes, en France comme au Maroc. 
Bien entendu, ceci ne vaut pas pour la plupart des croyants militants, à commencer par ceux qui ont pris pour cible le darwinisme chez les néoévangélistes américains comme chez certains musulmans (voir, par exemple, Yahya, 2006). L'opposition est ici frontale, vive, davantage argumentée. De tels individus manifestent un engagement fort, peuvent défendre des propositions susceptibles d’avoir un contenu un peu précis - même si celui-ci est totalement démenti par les faits. Et un tel militantisme a incontestablement une incidence politique et médiatique, en particulier sur les représentations du grand public. Simplement, ce que l'on veut souligner ici, c'est qu'il faut se garder de conclure, de l'existence de tels mouvements, que la thématique de l'origine de l'homme et des espèces vivantes contient, en substance, un motif nécessaire de conflit, qu'elle constitue une question par nature sensible et sacrée, y compris pour les croyants. Contrairement à ce que peut suggérer une interprétation trop rapide des vives polémiques qui entourent le darwinisme aux États-Unis, elle ne devient centrale que dans des circonstances historiques particulières, lorsque des groupes religieux parviennent à l'investir d'une forte puissance sacrée, à en faire une question aux implications morales et politiques majeures ${ }^{22}$. Les terrains que nous avons étudiés montrent que cette sacralité ne paraît pas nécessairement rattachée avec force à la conviction religieuse ordinaire. Du moins peut-elle n'avoir qu'un faible degré, la question n'occupant alors qu'une place très périphérique dans les préoccupations du croyant.

En d'autres termes, l'opposition frontale de certains mouvements créationnistes, chrétiens comme musulmans, avec le darwinisme, ne trouve sans doute pas sa source ultime et principale dans un donné d'ordre cognitif, dans la manière de se représenter la nature, son origine, et les êtres qui la peuplent. Elle n'est pas inscrite comme une nécessité dans le contraste entre des représentations du vivant qui seraient fondamentalement opposées chez ceux qui affichent une adhésion à la science, d’une part, et chez ceux qui affichent une adhésion à la religion, d’autre part. Il n’y a, sur ces thèmes, ni conflit, ni convergence absolument nécessaires, entre les croyants et les 
non-croyants ordinaires. Il y a un jeu complexe entre les représentations effectives de la nature vivante, leur perception croisée, leur étiquetage par les différents acteurs et le cadre historique dans lequel elles se déploient. Ce jeu ouvre un espace de croyances et d'interactions marqué par une large indétermination, laquelle tient pour beaucoup au fait que les représentations des mécanismes de production des espèces vivantes, dans le grand public, sont souvent éminemment flous et intéressent relativement peu dans le détail.

NDA. Les recherches évoquées dans cet article ont été réalisées avec le soutien des programmes ANR LICORNES (ANR-12-CULT-0002) et ASCE (ANR-13PDOC-0004). Je remercie François Ireton et Enrique Klaus pour m'avoir aidé dans la collecte et le traitement des données présentées dans la deuxième partie. Je remercie également le comité de rédaction de la revue et l'évaluateur de l'article pour leurs remarques et suggestions, dont je me suis efforcé de tenir compte.

\section{BIBLIOGRAPHIE}

BISHOP B. A. et ANDERSON C.W., 1990, «Student Conceptions of Natural Selection and Its Role in Evolution», Journal of Research in Science Teaching, 27, p. 415-428.

BOUDON R., 1992, L'Art de se persuader. Des idées douteuses, fragiles ou fausses, Paris, Seuil.

BRONNER G., 2007, «La résistance au darwinisme: croyance et raisonnement», Revue française de sociologie, 48-3, p. 587-607.

-, 2014, «Cognition et formation académique. Les professeurs de science de la vie et de la terre face au "problème des éléphants" 》, Revue européenne des sciences sociales, 52-I, p. I39-161.

DARWIN Ch., 1992 (1859), L'Origine des espèces, Paris, Garnier Flammarion.

DAWKINS R., 2006, The God Delusion, Boston, Houghton Mifflin.

-, 2009, Il était une fois nos ancêtres. Une histoire de l'évolution, Paris, Hachette.

DENNETT D., 2007, Breaking the Spell: Religion as a Natural Phenomenon, Londres, Penguin Books. 
DENIZ H., DONNELLY L. et YILMAZ I., 2008, «Exploring the Factors Related to Acceptance of Evolutionary Theory Among Turkish Preservice Biology Teachers », Journal of Research in Science Teaching, 45-4, p. 420-443.

EVANS E.M., 200I, «Cognitive and Contextual Factors in the Emergence of Diverse Belief Systems: Creation Versus Evolution», Cognitive Psychology, 42, p. 217-266.

GAYON J., 1992, Darwin et l'après-Darwin. Une histoire de l'hypothèse de sélection naturelle, Paris, Kimé.

GUILLO D., 2015, Darwin in France. Contributions to a Theory of Cognitive Sociology, Oxford, Bardwell.

FREUD S., 1985 (I9|I), «Une difficulté de la psychanalyse», in L'Inquiétante étrangeté, Paris, Plon.

KIM S. et NEHM R., 20II, «A Cross-Cultural Comparison of Korean and American Science Teachers Views of Evolution and the Nature of Science », International Journal of Science Education, 33-2, p. 197-227.

KONING D., 2006, «Anti-evolutionism Amongst Muslim Students», ISIM Review, vol. 18, p. 48-49.

LECOINTRE G. (éd.), 2009, Guide critique de l'évolution, Paris, Belin.

LECOURT D., 2007, L'Amérique entre la Bible et Darwin, Paris, PUF.

MAYR E., 200I, What Evolution Is, Londres, Penguin.

MEDIN D. et ATRAN S., 1999, Folkbiology, Cambridge, MIT Press.

PIETTE A., 2003, Le Fait religieux. Une théorie de la religion ordinaire, Paris, Economica.

STAVO-DEBAUGE J., 2012, Le Loup dans la bergerie. Le fondamentalisme chrétien à l'assaut de l'espace public, Genève, Labor et Fides.

YAHYA H., 2006, Atlas of Creation, Istanbul, Global publishing. 